VARIABILITE ACOUSTIQUE DES GROUPES DE CONSONNES : LE RÔLE DE LA VOYELLE ADJACENTE

\author{
C. MEUNIER \\ Institut de Phonétique, Université de Provence, 29 av. Robert Schuman, F-13621 Aix-en-Provence, \\ France
}

\begin{abstract}
The aim of this paper is to evaluate the influence of the vowel on the previous consonant cluster. We use the French Sounds Database (BDSONS) which proposes monosyllabic read words; we can thus opposite triple occurrences such as [pra]/[pri]/[pru]. We measure spectral material (F1 and F2) at center and 3/4 of the consonants duration. As we expected, the first consonant is less influenced than the second. But we note that the consonant itself and its acoustical specificities are an important factor to characterise the variation effects.
\end{abstract}

\title{
INTRODUCTION
}

Dans le cadre d'une étude sur la variabilité acoustique des groupes de consonnes du français, nous proposons d'évaluer le rôle de la voyelle sur les deux consonnes qui la précèdent. Nous concevons deux types de variables à prendre en compte: les variabless internes. d'une part, qui concernent les éléments constitutifs des GC (mode ou lieu d'articulation de l'une ou l'autre consonne du groupe, etc) et les variables externes, d'autre part, qui dépendent de facteurs non constitutifs des GC mais qui sont susceptibles d'entraîner des variations acoustiques à l'intérieur même du groupe (place dans le mot, place dans la syllabe, rôle de l'accent, type de prononciation, débit, voyelle adjacente, etc). Il n'est pas dans nos objectifs d'étudier l'ensemble des phénomènes de variabilité externe, la liste et trop longue, voire illimitée; nous en étudions cependant quelques uns supposés significatifs quant à la variation des GC. Nous nous intéressons ici précisément au rôle que joue la voyelle adjacente sur le spectre des consonnes du groupe (bémolisation ou "diésement" de la première, de la deuxième consonne ou des deux).

\section{MATERIEL. LiNGUISTIQUE}

Nous utilisons les corpus acoustiques de la Base de Données des Sons du français (BDSONS). Il s'agit des corpus $\mathrm{ACC} 01$ à ACC05, 5 corpus de 41 mots contenant chacun un groupe de consonnes, à partir desquels nous sélectionnons les GC suivis des voyelles cardinales /a/, /i/, /u/. Ces corpus ont été lu par 12 locuteurs, 6 femmes et 6 hommes. Nous tenons à préciser qu'il s'agit de lecture de mots isolés. L'ampleur et la diversité des corpus de BDSONS nous permettent de comparer un même groupe de consonnes en contact avec trois voyelles différentes (par exemple "flash", "flic", "flou"). Ce point est important car il permet d'évaluer précisément le rôle de la voyelle tout en neutralisant la variabilité interne au groupe de consonnes.

Le corpus est ainsi composé de mots monosyllabiques (la structure est de type $\mathrm{CCV}(\mathrm{C})$ ) contenant des groupes de deux consonnes. En première position on trouve des occlusives $([\mathrm{p}],[\mathrm{t}],[\mathrm{k}],[\mathrm{b}],[\mathrm{d}]$, $[\mathrm{g}]$,), des fricatives ([f], [s], [v], [z]) et des consonnes vocaliques ([1] et [r]), alors qu'en deuxieme position, les consonnes vocaliques ([1], $[\mathrm{r}],[\mathrm{w}])$ sont les seules présentes. Dans la plupart des cas la comparaison se fait sur des triplets: pra/prix/prou, slash/slip/sloop, etc; néanmoins, dans certains cas, le corpus ne nous a permis qu'une double comparaison: roi/rouis, wrappe/vrille, etc. 
Les groupes de consonnes sont au préalable étiquetés [1] et classés [2] de façon à cerner plus finement les effets de la voyelle en fonction des facteurs internes de variation des groupes de consonnes (lieu d'articulation, mode d'articulation, mode de phonation).

\section{ANALYSE ACOUSTIQUe}

L'analyse acoustique porte sur les mesures spectrales de $\mathrm{C} 1$, de $\mathrm{C} 2$ et de la voyelle (mesure de $\mathrm{F} 1$ et F2): pour C2, les spectres sont mesurés au centre et aux 3/4 des consonnes afin d'observer si l'influence de la voyelle porte sur l'ensemble de la consonne ou seulement sur la zone transitoire vers la voyelle; pour $\mathrm{Cl}$, il faut différencier les types de consonnes: on mesure la fréquence du bruit d'explosion pour les occlusives et la fréquence du formant de bruit pour les constrictives (pic le plus haut sur le spectre au centre et au $3 / 4$ de la durée de la fricative); les consonnes vocaliques sont mesurées de façon identique à celle de C2. Enfin, on mesure également le spectre du centre de la zone stable de la voyelle afin de contrôler l'homogénéité de chacune des trois classes de voyelle et de comparer ces données à celles des consonnes. Les analyses acoustiques ont été effectuées à l'aide du logiciel PTS développé à l'I.C.P. de Grenoble [3].

\section{HYPOTHESES ET QUESTIONS PRELIMINAIRES}

Certains travaux ont déjà montré que les caractéristiques acoustiques des consonnes vocaliques sont dépendantes des voyelles adjacentes [4] et [5]. On s'attend donc à ce que C2 subisse plus fortement l'influence de la voyelle que $\mathrm{C} 1$. On peut cependant s'interroger sur cette "résistance" de $\mathrm{C} 1$ : est-elle due à cette place (C1) plus éloignée de la voyelle, ou bien à la "nature" de la consonne (C1 est habituellement une occlusive ou une fricative; ne s'agit-il pas là de consonnes plus résistantes à l'assimilation que les consonnes vocaliques)?

Nous nous interrogeons également sur l'homogénéité des classes de consonnes. Les consonnes subissent-elles la même influence à l'intérieur de chaque classe (occlusives, fricatives, et consonnes vocaliques) ou varient-elles indépendamment de leur classes? Dans la mesure où le facteur de variabilité que l'on fait jouer est le lieu d'articulation (déplacement de zones spectrales), on peut s'attendre à ce que les consonnes aient un comportement différent en fonction de leur lieu d'articulation et non en fonction des classes de consonnes que nous avons définies, fondées sur le mode d'articulation.

\section{RESULTATS}

\section{Deuxieme consonne:}

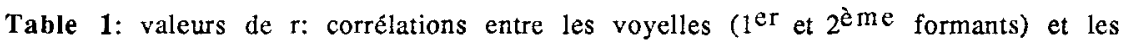
consonnes $\mathrm{C} 2$ (au centre " $c$ " et au $3 / 4$ de la durée de la consonne)

\begin{tabular}{|l|c|c|c|c|c|c|c|c|c|}
\cline { 2 - 10 } \multicolumn{1}{c|}{} & \multicolumn{2}{c|}{$\mathrm{C}$} & $3 / 4$ & \multicolumn{2}{c|}{$\mathrm{C}^{\mathrm{nvs-r}}$} & $3 / 4$ & voc1 & bat & \multicolumn{2}{c|}{ voc2 } & \multicolumn{2}{c|}{ C } & $3 / 4$ \\
\hline $\begin{array}{l}\text { F1 } \\
\text { Voy }\end{array}$ & 0,549 & 0,795 & $-0,103$ & 0,14 & 0,135 & 1,96 & 0,444 & 0,209 & 0,678 \\
\hline $\begin{array}{l}\text { F2 } \\
\text { Voy }\end{array}$ & 0,496 & 0,785 & 0,387 & 0,799 & 0,534 & 0,544 & 8,868 & 0,293 & 0,695 \\
\hline
\end{tabular}

Ainsi que nous l'attendions, C2 subit plus fortement l'influence de la voyelle. Cependant, cette influence ne se manifeste pas de la même façon pour toutes les consonnes vocaliques.

[1] est affecté dans son ensemble, aussi bien pour F1 que pour F2 et au centre comme au 3/4, même si les valeurs de F1 et de F2 se rapprochent plus précisément de la voyelle adjacente au $3 / 4$ (voir table 1 , figures 2,3 ). 


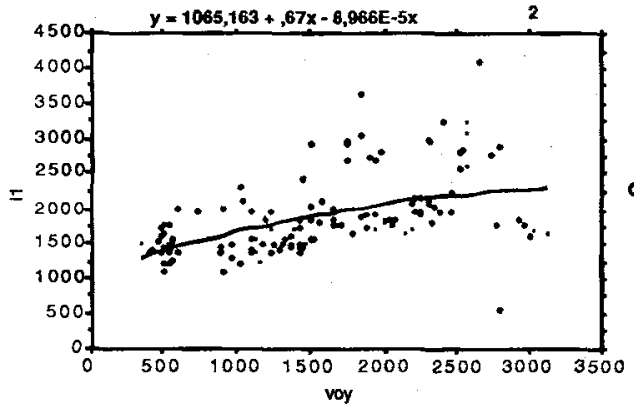

Figure 2: régression polynomiale: en abscisse le F2 des voyelles [a], [i], [u], en ordonnée les valeurs de F2 de [l] prise au centre de la consonne

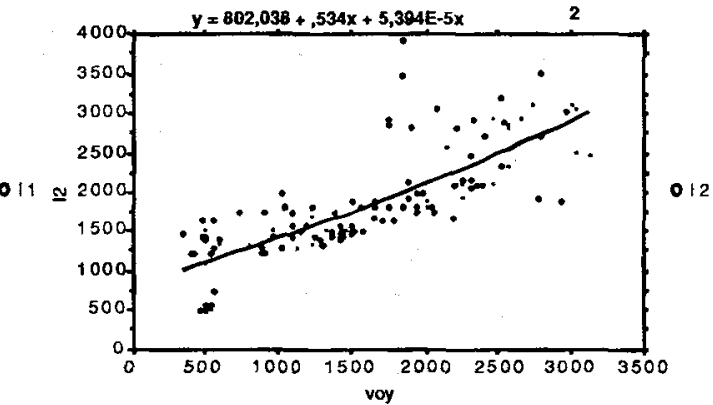

Figure 3: régression polynomiale: en abscisse le F2 des voyelles [a], [i], [u], en ordonnée les valeurs de F2 de [l] prise au $3 / 4$ de la consonne

Le [r], qu'il soit en contexte voisé ou non, est affecté essentiellement sur le deuxième formant, alors que le premier formant ne semble pas évoluer de façon similaire à la voyelle. En ce qui concerne la première partie vocalique du [r] vocalique à battement, le F2 semble lui aussi affecté par la voyelle (voir table 1, figures 4,5 ).

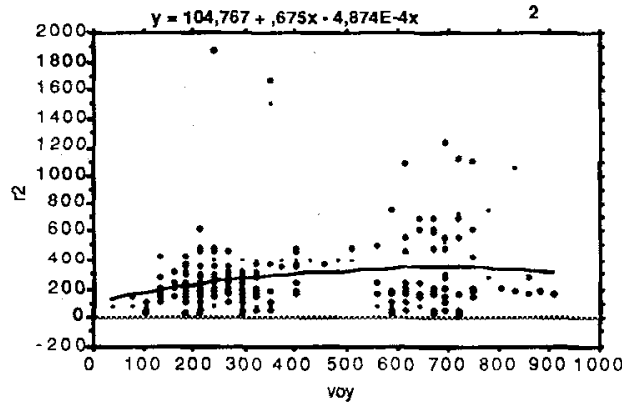

Figure 4: régression polynomiale: en abscisse le $\mathrm{E} 1$ des voyelles [a], [i], [u], en ordonnée les valeurs de F1 de [r] prise sur la deuxième partie vocalique.

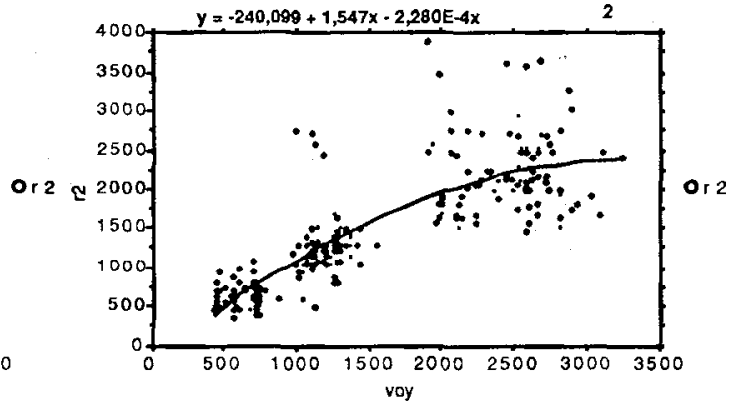

Figure 5: régression polynomiale: en abscisse le E2 des voyelles [a], [i], [u], en ordonnée les valeurs de F2 de [r] prise sur la deuxième partie vocalique.

Inversement, la glissante $[\mathrm{w}]$ subit l'influence de la voyelle au $3 / 4$ et non au centre, que ce soit pour F1 ou F2 (voir table 1, figures 6,7 ).

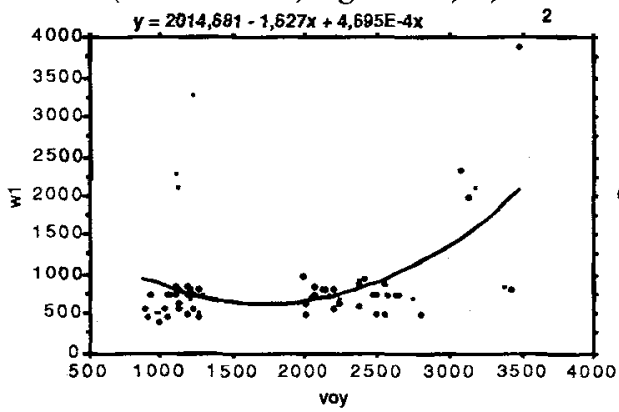

Figure 6: régression polynomiale: en abscisse le F2 des voyelles [a], [i], [u], en ordonnée les valeurs de F2 de [w] prise au centre de la consonne

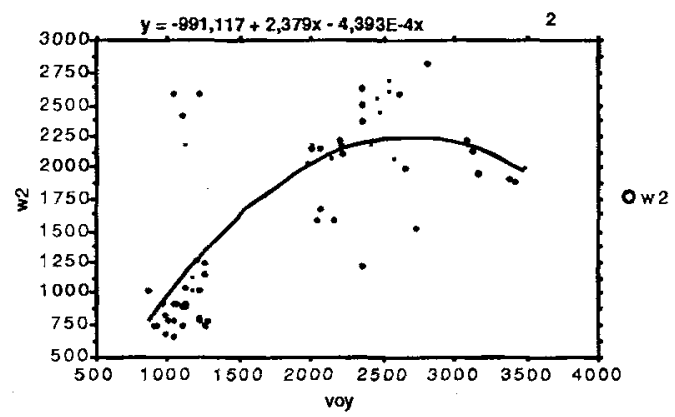

Figure 7: régression polynomiale: en abscisse le F2 des voyelles [a], [i], [u], en ordonnée les valeurs de F2 de [w] prise au $3 / 4 d \theta$ la consonne

\section{Première consonne}

En ce qui concerne la première consonne du groupe, l'influence de la voyelle est très nettement atténuée. En fait, comme pour C2, la variation dépend du type de consonne. 
Nous n'avons noté aucune corrélation significative entre les fricatives, quelles qu'elles soient, et la voyelle adjacente. Ce type de consonne, en première position, semble totalement résistant à l'influence de la voyelle.

En ce qui concerne les occlusives, seules les vélaires sont significativement corrélées aux voyelles adjacentes: la valeur $\mathrm{r}$ de corrélation entre les voyelles et [k], [g] et de 0,431 , ce qui, pour 67 occurences est significativement positif au seuil de 0,02. Ce résultat est intéressant car il montre que les vélaires, dont les transitions et le bruit d'explosion sont dépendants de la voyelle adjacente en contexte $\mathrm{CV}$, continuent à subir cette influence lorsqu'elles sont séparées de la voyelle par une autre consonne.

Les deux consonnes vocaliques en position $\mathrm{Cl}$ ont un comportement différent: si [r] résiste à l'influence de la voyelle ( $r=0,156$, non significatif, pour $F 2$, au 3/4), [1] varie significativement en fonction de la voyelle $(r=0,536$ significatif à un seuil de 0,02 , pour $F 2$, au 3/4) suivante:

On peut résumer l'influence de la voyelle sur le groupe de consonnes qui la précède de la façon

a) Dans un $\mathrm{GC}$ de structure $\mathrm{C}_{1} \mathrm{C}_{2} \mathrm{~V}, \mathrm{C} 1$ subit une variation plus importante que $\mathrm{C} 2$ (la corrélation $[1] /$ voyelle est plus significative lorsque [l] est en seconde position).

b) La variation de la consonne est fonction de son lieu d'articulation (les vélaires sont significativement influencées par la voyelle alors que les autres occlusives ne sont pas affectées) et de son mode d'articulation (les fricatives semblent totalement résistantes à l'influence de la voyelle, alors qu'occlusives et consonnes vocaliques sont partiellement affectées).

c) Le caractère transitoire ou stable des consonnes les rend "résistantes" ou "influençables" (au centre de la glissante [w] la consonne tend vers les caractéristiques acoustiques de [u], résistant ainsi à l'influence des autres voyelles; la consonne [1], la plus stable acoustiquement, subit l'influence de la voyelle dès le centre).

d) Les écarts types importants observés dans les moyennes spectrales de F1 et F2 de chaque consonne ne sont pas forcément dus à une forte variabilité mais à des stratégies différentes des locuteurs (la premiere partie vocalique de [r] lorsqu'il est battu est, soit une "pré-production" de la voyelle suivante, [ $\mathrm{b}_{\mathrm{a}}$ ra], soit la réalisation d'un élément vocalique neutre [bəra], et ceci semble dépendre de la statégie que le locuteur utilise[6]).

\section{CONCLuSION}

Il est délicat de parler des groupes de consonnes comme d'un ensemble homogène. Une consonne est souvent définies par défaut; elle est avant tout "non voyelle". Il semble difficile de trouver un indice acoustique commun à toutes les consonnes telles que la stabilité et les formants caractérisant les voyelles. Cette étude le montre une fois de plus, les consonnes ont des caractéristiques acoustiques très diverses et se comportent très différemment face à un même facteur de variabilité. Ces spécificités sont à prendre en compte dés lors que l'on étudie les consonnes dans leur ensemble. L'objectif de notre travail est d'évaluer la variabilité (causes) et les caractéristiques de la variation (conséquences), afin de cerner plus précisément la spécificité et les particularités du consonantisme.

\section{REFERENCES:}

[1] AUTESSERRE, D., ROSSI, M., 1989, "Un méthode de segmentation et d'étiquetage des groupes consonantiques de la base de données des sons du français", Journal d'Acoustique, 2, 31 1-322.

[2] MEUNIER, C.,1990, "Groupes consonantiques: premier inventaire des réalisations acoustiques des phases de transition", Actes des 18èmes Journées d'Etude sur la Parole, Montréal, p. 69-73

[3] CAEROUX, J.C., DOLMAZON, J.M., 1990, PTS Software V. 4.21, User Manual, Document du Projet Esprit SAM 2589.

[4] CHAFCOULOFF, M., 1979, "Les propriétés acoustiques de [j, y, w, 1, r] en français", Travaux de l'Institut de Phonétique d'Aix, 6, 10-24.

[5] CHAFCOULOFF, M., 1980, "Les caractéristiques acoustiques de /j, w, 1, r/ en français", Travaux de l'Intitut de Phonétique d'Aix, 7, 7-56.

[6] MEUNIER, C., AUTESSERRE, D.,1989, "Variabilité intra et inter individuelle de l'allophone vocalique de /r/ en contexte occlusif voisé", Actes du séminaire Variabilité et spécificité du locuteur: études et applications, Marseille, H. Méloni (eds), p.36-39. 